\section{LOOK, NO HANDS!}

Dürr Dental's new ‘Touchless' contact-free soap and disinfectant dispenser combines good looks with impressive functionality. The failsafe automatic sensor reacts as soon as your skin comes within its detection range, dosing exactly the right dosage of liquid. It can be used for disinfectant, liquid soap or hand lotion. Its aluminium, anodised casing hinders the growth of bacteria by virtue of its bacteriostatic effect.

Not only is it easy to use, it's also easy to clean. Thanks to a 'push and click' system and a pivoting discharge plate, the stainless steel pump can be removed from the front and placed back in later on without the need to remove the dispenser from the wall. Five replaceable single cell batteries, which last up to two years, ensure a long and reliable service life. An audible signal indicates the need for a battery change.

This new dispenser is part of the reliable range of hygiene products from Dürr Dental. For more information visit www.duerrdental.com.

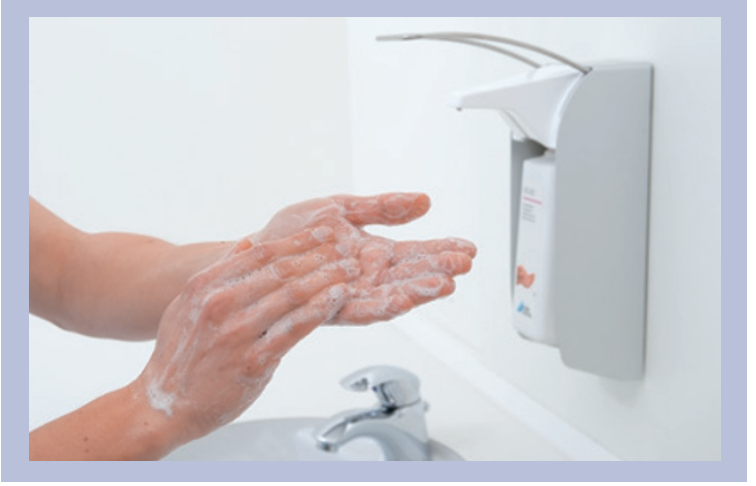

\section{PAIN FREE BIOFILM MANAGEMENT}

The HELBO-Therapy is a clinically proven photodynamic procedure, which combats bacterial infections in the oral cavity. Many users have been employing this therapy for a number of years and value it highly.

The HELBO-Therapy reduces infection-causing pathogenic bacteria by over 99\% and completely without side effects. In this procedure, which has been scientifically researched and documented in a variety of indications for more than twelve years, the microorganisms are first stained (HELBO Blue Photosensitiser) and finally exposed under the HELBO TheraLite Laser, a non-thermally acting laser. The laser light forms highly reactive singlet oxygen which leads to lipid oxidation of the bacteria's membrane, thus destroying pathogenic bacteria in the shortest possible time-frame. The procedure is both pain and side effect free. Over 70 publications document evidence in support of this therapy. Now, in addition to this, the DVK has issued a recommendation for the reimbursement of treatments for biofilm-associated infections of the oral cavity (periodontology, peri-implantitis therapy), if certain conditions are fulfilled.

Information on HELBO therapy is available at: www. helbo.de.

\section{NEW WEBSITE LAUNCHED}

The British Dental industry Association (BDIA) has announced the launch of a completely redesigned version of its website, www.bdia.org.uk.

Key features of the site include a refreshed and more impactful look, a more engaging user experience for both BDIA members and the dental team with enhanced content, navigation and new functionality. All this together with greater optimisation for mobile devices will allow both BDIA members and the wider dental profession to interact more easily with the Association online.

A number of practical enhancements have been made including:

- Clear messaging for different audiences including members, the profession and non-members whilst effectively informing the whole sector of the key activities upon which the BDIA is focused

- New site navigation and more prominent search functionality making it quick and easy for the dental profession to connect with quality-focused BDIA members

- New interface enabling BDIA members to easily update their own company profile including changes to their products, ensuring the whole dental team has access to the very latest information

- New application form enables companies wishing to join the BDIA with an option to do so online

- 'At a glance' calendar of BDIA and wider industry events

- Exclusive password protected BDIA Members Area provides quick and easy access to valuable resources to help keep members fully up to date with the latest industry news, statistical research, technical and regulatory updates, expert advice and more.

To see the new-look website visit www.bdia.org.uk.

\title{
NOW EVEN MORE SUPPORT FOR YOUR PRACTICE
}

In response to client feedback, DPAS Dental Plans is delighted to launch 'Business Bites', a range of practical business solutions and special offers designed to support practice growth.

Available exclusively to DPAS clients, Business Bites is the latest initiative from DPAS Solutions and provides practices with all the convenience of a 'one stop shop', but with the flexibility to choose the products and services that best suit their needs.

Through Business Bites, DPAS has teamed up with some of the dental industry's leading providers, offering their clients an exclusive selection of special offers from the likes of ADAM (Association of Dental Administrators and Managers), Apolline, Carefree Credit Dental, Dental Focus, Dentally, 4dentists group, Lloyds Bank Cardnet, Medifinance, Milkshake Marketing, Practices Made Perfect and ProDentalCPD.

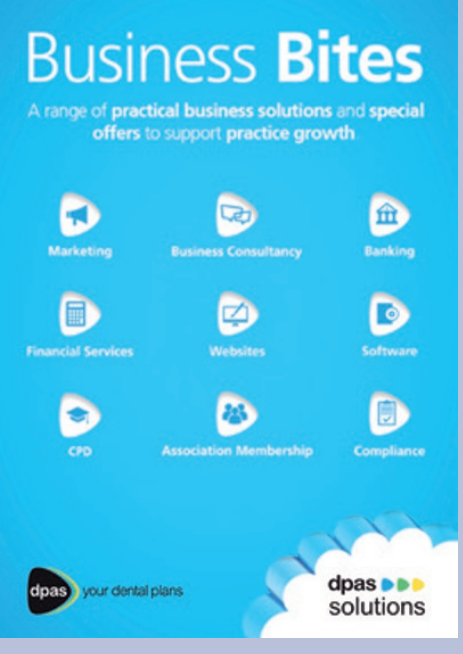

As experts in dental plan administration, DPAS believe practices deserve expert support in all areas of their business. Remaining absolutely focused on providing comprehensive dental plan support services and offering the best value in the marketplace, DPAS has now enlisted trusted partners to offer their clients more support than ever before. 